OPEN

SUBJECT AREAS: APPLIED MATHEMATICS

PHYSICAL SCIENCES

Received

11 October 2013

Accepted

7 November 2013

Published

27 November 2013

Correspondence and requests for materials should be addressed to

J.A.C.G. (jason. gallas@cbi.unierlangen.de)

\section{Discontinuous Spirals of Stable Periodic Oscillations}

\author{
Achim Sack' , Joana G. Freire ${ }^{1,2,3}$, Erik Lindberg ${ }^{4}$, Thorsten Pöschel ${ }^{1,2}$ \& Jason A. C. Gallas ${ }^{1,2,3,5}$
}

${ }^{1}$ Institute for Multiscale Simulations, Friedrich-Alexander Universität, D-91052 Erlangen, Germany, ${ }^{2}$ Departamento de Física, Universidade Federal da Paraíba, 5805 1-970 João Pessoa, Brazil, ${ }^{3}$ Centro de Estruturas Lineares e Combinatórias, Faculdade de Ciências, Universidade de Lisboa, 1649 -003 Lisboa, Portugal, ${ }^{4}$ DTU Elektro Department, 348 Technical University of Denmark, DK2800 Lyngby, Denmark, ${ }^{5}$ Instituto de Altos Estudos da Paraíba, Rua Infante Dom Henrique 100-1801, 58039-150 João Pessoa, Brazil.

We report the experimental discovery of a remarkable organization of the set of self-generated periodic oscillations in the parameter space of a nonlinear electronic circuit. When control parameters are suitably tuned, the wave pattern complexity of the periodic oscillations is found to increase orderly without bound. Such complex patterns emerge forming self-similar discontinuous phases that combine in an artful way to produce large discontinuous spirals of stability. This unanticipated discrete accumulation of stability phases was detected experimentally and numerically in a Duffing-like proxy specially designed to bypass noisy spectra conspicuously present in driven oscillators. Discontinuous spirals organize the dynamics over extended parameter intervals around a focal point. They are useful to optimize locking into desired oscillatory modes and to control complex systems. The organization of oscillations into discontinuous spirals is expected to be generic for a class of nonlinear oscillators.

F ully characterizing self-oscillations in nonlinear oscillators demands knowledge both of its phase-space dynamics, and the corresponding dual dynamics seen in stability (phase) diagrams in the system control parameter space. While phase-space dynamics has been extensively studied ever since the pioneering works of Poincaré and others well over a century ago, the associated description of stability diagrams had to wait for the advent of modern computers, particularly to characterize the intricate chaotic phases and complex phases corresponding to periodic motions other than stationary or the simple nonstationary periodic oscillations which appear immediately after Hopf bifurcations. Although it is not difficult to calculate Hopf boundary curves, no prescription exist for delimiting stability domains of the large succession of oscillatory phases that normally follow Hopf bifurcations.

For a number of years, the study of bifurcation boundaries was conducted mainly for systems governed by maps, since they are much easier to deal with both analytically and computationally. Bifurcation boundaries in phase diagrams computed for maps were discussed recently by Lorenz in what turned out to be his last paper ${ }^{1}$. But the situation is evolving fast. Presently, detailed stability diagrams resulting from extensive simulations are becoming available not only for maps but also for a number of flows, i.e. for systems governed either by finite sets of ordinary nonlinear differential equation $s^{2-8}$ and, more recently, for infinite dimensional systems controlled by delay-differential equations, such as semiconductor lasers with delayed feedback'.

Experimentally, the determination of stability boundaries beyond the Hopf lines is a very difficult problem. This is the challenge that we wish to address here. More specifically, we report experimental phase diagrams of high-resolution displaying detailed information about the stability boundaries measured for an electronic circuit. Such stability diagrams summarize the dynamics over extended intervals of control parameters and provide detailed information about virtually all oscillations present in the circuit, including chaotic ones. Our diagrams reveal an unanticipated and quite surprising organization of parameters, that form a large discontinuous spiral of the stability phases underlying the set of periodic oscillations. Whirling along the discontinuous phases of the spiral implies having to jump repeatedly between an infinite alternation of chaotic and periodic phases. As explained below, such discontinuities are signatures of systematic bifurcation cascades unfolding according to a hitherto unseen and more complex scenario not included, e.g. in the famous classification of Arnold ${ }^{10}$. Since all oscillations underlying the discontinuous spiral are stable oscillations, the spiral is directly accessible to experimental work.

From a theoretical point of view, the determination of the frontiers between chaotic and periodic phases of oscillators is a complex task that needs to be addressed in all branches of the natural sciences. Unlike the relatively 
tame situation for fixed equilibria, whose boundaries are accessible by straightforward calculations, not even approximate methods exist for delimiting parameter windows likely to contain, e.g. chaotic bursting or pulsation phases of high-periodicity and their rich accumulation limits which are so typical in laser dynamics ${ }^{11}$, in biological neural networks ${ }^{12}$, and in many other applications ${ }^{13}$. Even worse, it seems likely that no analytical methods will ever exist to delimit such stability domains. For instance, think of the problem of computing analytically the self-similar boundary of the Mandelbrot set. Thus, the theoretical delimitation of stability phases can be dealt numerically only. Regularities of complex phases are universal invariants that help unify seemingly distinct systems into universality classes whose knowledge is a great asset for applications.

\section{Results}

Our experiments are conducted with the help of the electronic circuit shown schematically in Fig. [1]. The circuit is a slight variation of an autonomous Duffing-like proxy introduced recently ${ }^{14,15}$, having the distinctive technological advantage of bypassing noisy spectra which normally pollute driven (i.e. non-autonomous) oscillators. An additional characteristic is that our oscillator is among the most precise devices presently in existence, allowing its modes of oscillation to be measured with very high accuracy. In a variety of configurations, such oscillators are also popular nowadays as components in experiments conceived to study the variegated complex phenomena predicted for nonlinear oscillators. Our circuit is governed by the equations

$$
\ddot{x}-b \dot{x}-x+x^{3}+k z=0, \quad \dot{z}=(\dot{x}-z) \omega,
$$

where variables and parameters are all adimensional ${ }^{14,15}$. Equivalently, these equations can be also conveniently written as

$$
\dot{x}=y, \quad \dot{y}=x-x^{3}+b y-k z, \quad \dot{z}=(y-z) \omega .
$$

Here, $b$ is used to regulate the intensity of the damping, $k$ is the feedback amplitude and $\omega$ is the cut-off frequency of the filter ${ }^{14,15}$.
Essentially, $b$ and $k$ are factors of the voltage dividers in the circuit. Instead of the external sinusoidal drive commonly used in the standard Duffing oscillator, usually defined as $\ddot{x}+b \dot{x}-x+x^{3}=a$ $\sin (\omega t+\varphi)$, our circuit has an additional degree of freedom, $z$, describing the inertia of the low-pass filter. Noteworthy is the sign of the damping term $b$, opposite to the sign normally present in the standard oscillator. As indicated in Fig. [1], the Duffing-like proxy allows one to adjust both parameters $b$ and $k$ independently, by means of digitally controlled attenuators. In addition, the original nonlinearity formed by two anti-parallel diodes ${ }^{14,15}$ was replaced here by the diode network, also shown in Fig. [1], used to produce the cubic nonlinearity of the circuit. The replacement of the two antiparallel diodes turned out to be crucial to obtain a reasonable agreement between the circuit and its model equations above.

Figure [2] illustrates five typical wave patterns $x(t)$ obtained numerically for our circuit, together with their respective return maps, as indicated in the figure caption. The patterns labeled $A, B$, $C$ display multipeaked periodic oscillations while patterns $a$ and $b$ illustrate chaotic oscillations. Patterns $A, B, C$ show clearly that the Duffing-like proxy displays precisely the same antiperiodic oscillations that were discovered recently in a different experiment ${ }^{4}$. In contrast with that earlier work, which is governed by four differential equations, the Duffing-like proxy generates antiperiodicity in a considerably simpler setup, involving just three equations. In panels $A, B$, $C$ of Fig. [2] we also compare numerically computed with experimentally measured return maps (shown in red) for the maxima in the $x(t)$ variable: $x\left(t_{\max }\right) \times x\left(t_{\max +1}\right)$. They illustrate the very good agreement obtained between measurements and simulations. Similar agreement is found for the other variables (not shown). The periodic waveforms $A, B, C$ were obtained for $(b, k)=(0.3646,2.114),(0.3481$, $1.997)$ and $(0.3373,1.914)$, and have periods $T_{A}=42.696, T_{B}=$ 50.175 , and $T_{C}=57.924 \mathrm{~ms}$, respectively. The corresponding experimental points are $(b, k)=(0.4906,1.976),(0.4766,1.892)$, and $(0.4669,1.804)$. The waveforms labeled $a$ and $b$ illustrate non-periodic (chaotic) oscillations obtained for $(b, k)=(0.356,2.05)$ and
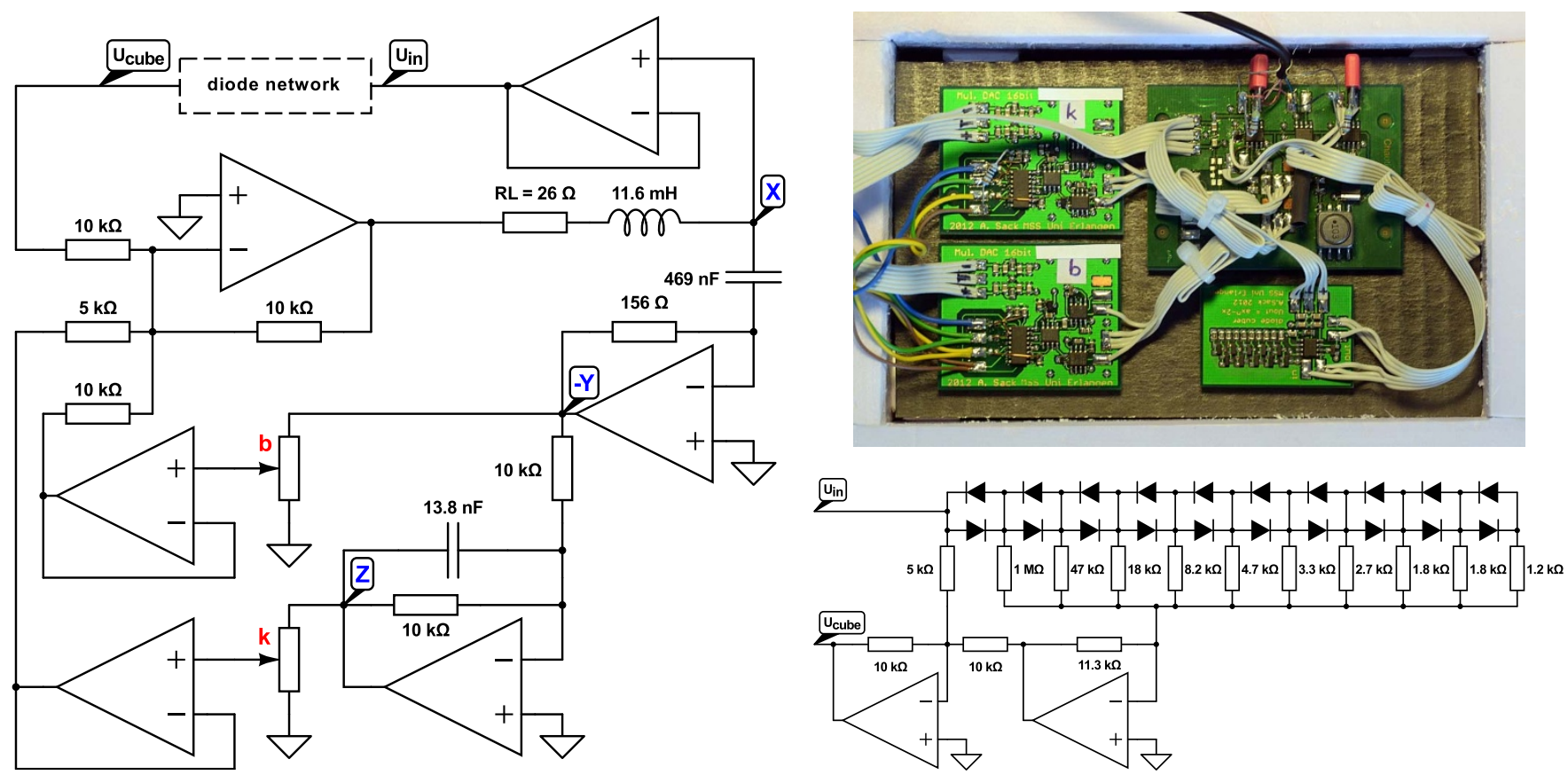

Figure 1 Schematic representation and a photograph of the circuit used to produce the discontinuous spiral shown in Fig. [3](a). The flow governing this circuit is defined by Eq. (2). Note how parameters $b$ and $k$ are tuned and the definitions of the dynamical variables $x, y, z$ used in Eq. (2). Also shown is the "diode network" inside the dashed box seen in diagram on the left. Measurements are robust against variations of the nominal parameter values. 

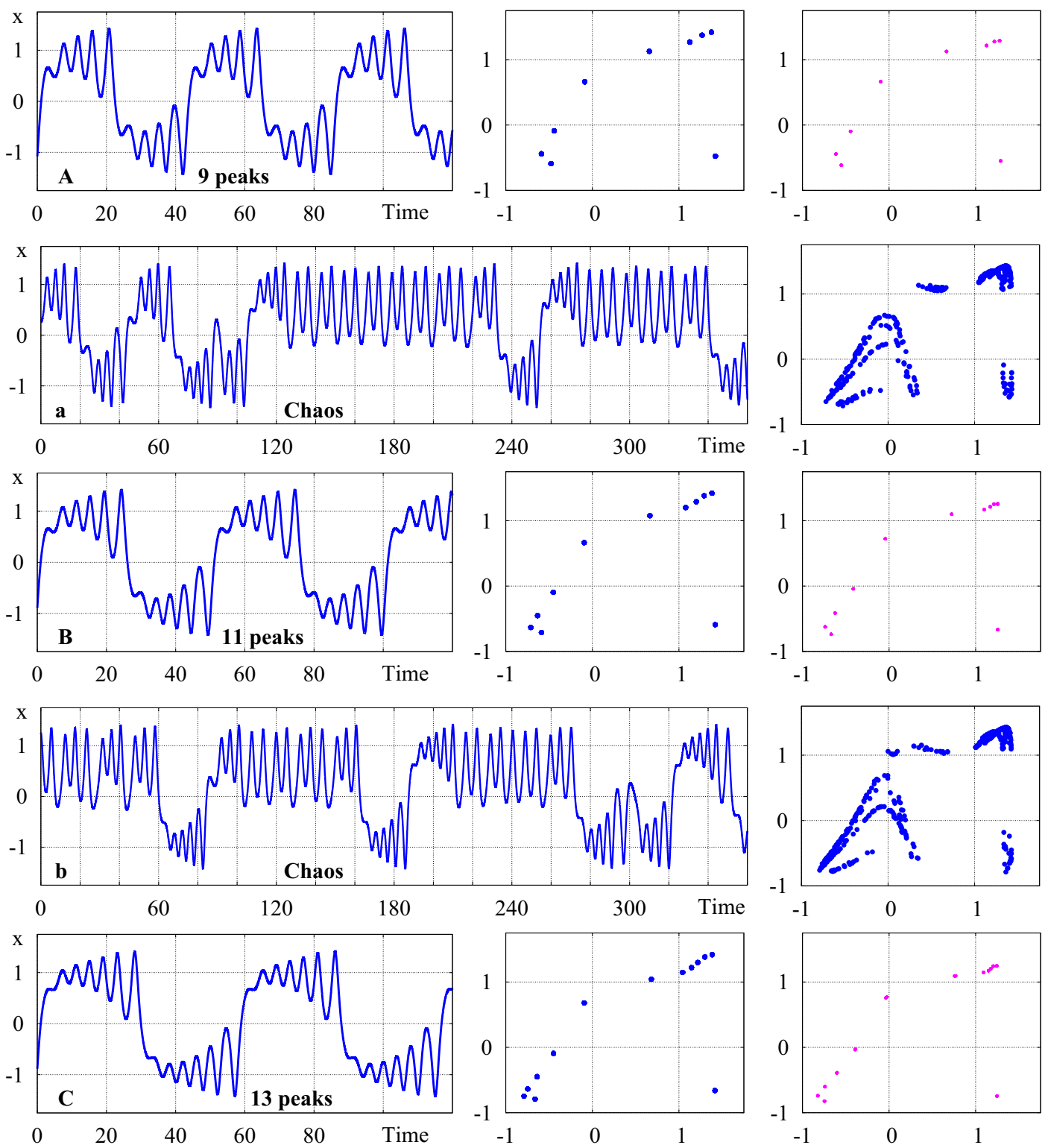

Figure $2 \mid$ The leftmost panels show typical time-evolutions of periodic and aperiodic ("chaotic") waveforms $x(t)$. The labels $A, B$ and $C$ refer to parameters indicated by dots inside the fish-like structures in Fig. [5] (a), and correspond to antiperiodic oscillations $s^{4}$. The labels $a$ and $b$, corresponding to points $a$ and $b$ shown in Fig. [5] (a), refer to chaotic oscillations. The rightmost panels in $A, B$ and $C$ show (in red) experimental return maps $x\left(t_{\text {max }}\right) \times$ $x\left(t_{\max +1}\right)$, while all other (blue) panels refer to numerically obtained time evolutions of $x(t)$ and their corresponding return maps. Time is measured in ms.

$(0.342,1.95)$, respectively. The location in parameter space of all points $A, a, B, b, C$ is indicated in Fig. [5](a) and serve to indicate how the oscillations unfold when moving towards the focal point.

The regularity observed for $A, B$, and $C$ in Fig. [2] prompts a natural question: How do such oscillations organize themselves in control parameter space when parameters are tuned continuously? To answer this question, we performed a very detailed global investigation of the phase-space of the system for a wide interval of parameters. More specifically, for all representative combinations of parameters and initial conditions over wide intervals, we conducted a two-phase experiment as follows: First, we discriminated whether oscillations are chaotic or periodic and then, second, recorded this information on a fine grid $(b, k)$ of parameters, to produce phase diagrams as described in details below, in Methods. A typical example of an experimentally obtained phase diagram is shown in Fig. [3](a). This figure records how much the oscillations spread in phase-space, i.e. it records how the occupied fraction of the phase-space varies as a function of $b$ and $k$. The total time needed to collect the experimental data summarized in Fig. [3](a) was $\sim 31$ days, a quite long time that emphasizes the importance of the thermal bath that was used during data acquisition (see Methods).

The measurements collected in the phase diagram shown in Fig. [3] (a) display our key result: the discovery of a wide discontinuous spiral structure. The spiral is formed by an alternation of stability phases arising from standard saddle-node bifurcations with endpoints (terminations) as shown magnified in Fig. [4]. The existence of spirals and exceptional points organizing oscillations in the control space of a number of systems was reported in the last few years, e.g. in tunnel diodes and fiber-ring lasers ${ }^{2}$, electronic circuits ${ }^{3,5}$, for semiconductor lasers, for light-emitting diodes with optoelectronic feedback, in chemical and biochemical oscillators, in tunnel diodes, and in a few other paradigmatic flows ${ }^{2-9,16,17}$. However, all studies done so far have consistently reported the observation of continuous spirals formed by shrimps ${ }^{1}$. The novel spiral reported here, shown in 

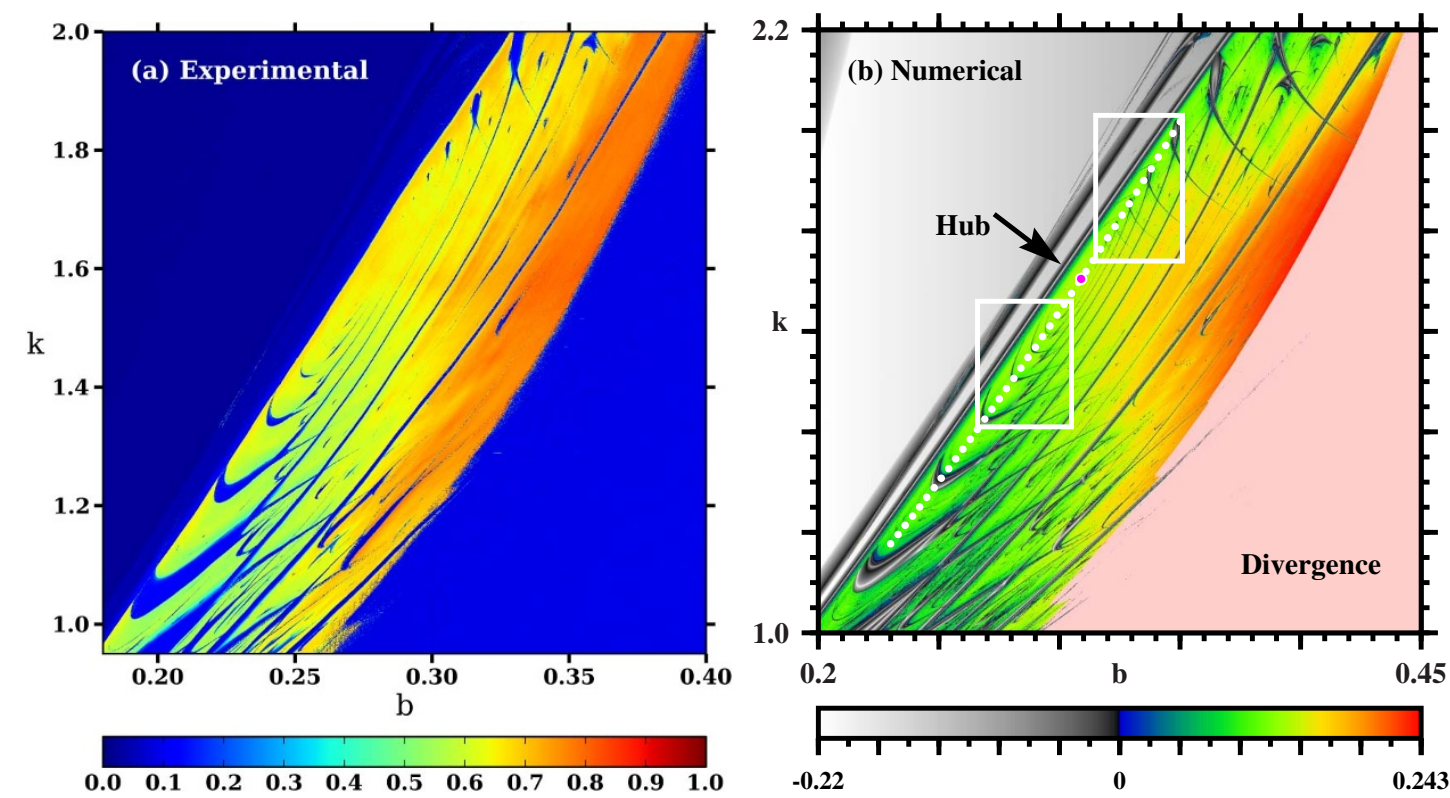

Figure 3 Comparison between discontinuous spirals obtained (a) experimentally and (b) numerically. (a) Experimental stability diagram displaying $1300 \times 2048$ individual measurements. (b) Lyapunov stability diagram showing $2400 \times 2400$ exponents. The arrow points to the focal hub (red dot), located at $(b, k) \simeq(0.309,1.7046)$. The dotted line represents a fit passing through the widest periodicity islands near the hub. The turning points inside the white boxes are shown magnified in Fig. [4]. The slight differences in scales are explained in the text.

Fig. [3], is neither continuous nor composed of shrimps but, instead, of rather distinct terminations, to be described below.

An important fact about Fig. [3] (a) is that it displays a lot of details about the global structure of the control space despite the fact that both methods used to record our data contain no objective rule to distinguish between chaos and periodicity of the oscillations. Thus, one may ask: What is the meaning of all the information gathered experimentally in Fig. [3](a)? The answer can be obtained by resorting to the three differential equations defined by the circuit. With them, we computed Lyapunov stability diagrams as described in Methods, at the end of the paper. Such Lyapunov exponents are standard tools used to discriminate between chaos and periodicity for every parameter pair $(b, k)$.

Figure [3](b) shows the largest non-zero Lyapunov exponent and illustrates how periodic and chaotic solutions self-organize over a wide region of the control parameter space for $\omega=0.5$, an arbitrary choice motivated by the original experiments of Lindberg et al. ${ }^{14,15}$. Results are not sensitive to this choice. As shown by the colorbar in Fig. [3](b), darker shadings are used to denote periodic oscillations

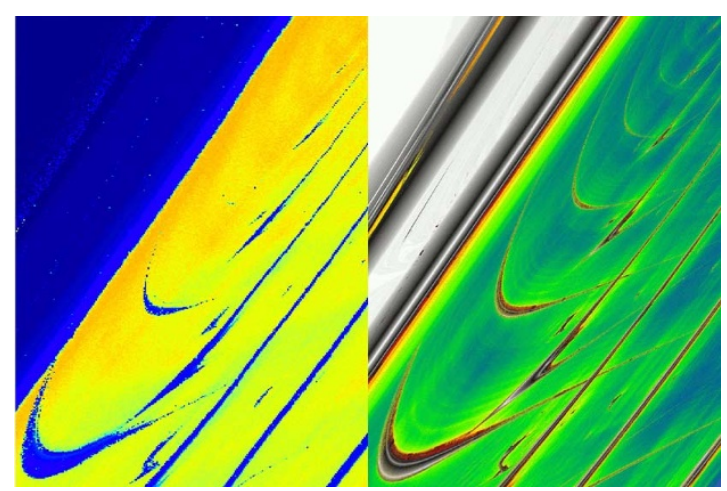

(a) (i.e. negative Lyapunov exponents) while colors represent chaotic phases (positive exponents). Similarly to the experimental phase diagram of Fig. [3](a), the Lyapunov phase diagram in Fig. [3](b) also displays the spiral formed by a discontinuous alternation of stability phases. In this figure we have indicated the accumulation focal point, hub, to where all phases of periodic oscillations seem to converge. The dotted line is a fit to points centered at the terminations of the several periodic phases. It is defined by the equation $k=$ $k(b)=7.98844 b^{2}+2.37172 b+0.209081$. The focal hub is located on this line, indicated by a red dot. The two white boxes show the systematic way in which the self-similar endpoints (terminations) of each individual periodic phase accumulates towards the focal hub. The detailed structures inside such boxes are shown magnified in Fig. [4], both for the experimental and numerical results.

Since the Lyapunov exponent is the standard quantitative criterion to distinguish between chaos and periodicity, Fig. [3](b) validates the procedure used to represent the experimental data in Fig. [3](a). Furthermore, the diagrams in Fig. [3] clearly show that our algorithms to deal with experimental data are capable of discriminating

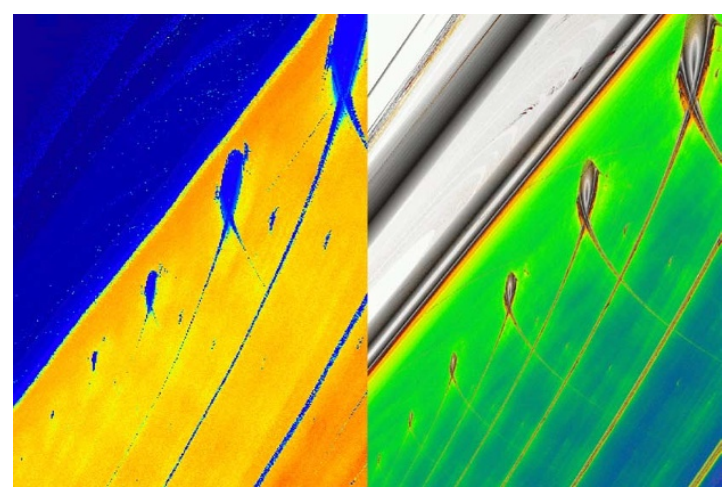

(b)

Figure 4 Enlarged views of the two sets of terminations composing the discontinuous spiral. (a) Comparison between experimental and numerical boomerang- and cusp-like terminations from the leftmost white box in Fig. [3](b). (b) Comparison of the "fish-like" terminations seen in rightmost white box in Fig. [3](b). Cusps are the terminations of the very long left fins of the fishes. The color tables are the same ones used in Fig. [3]. 

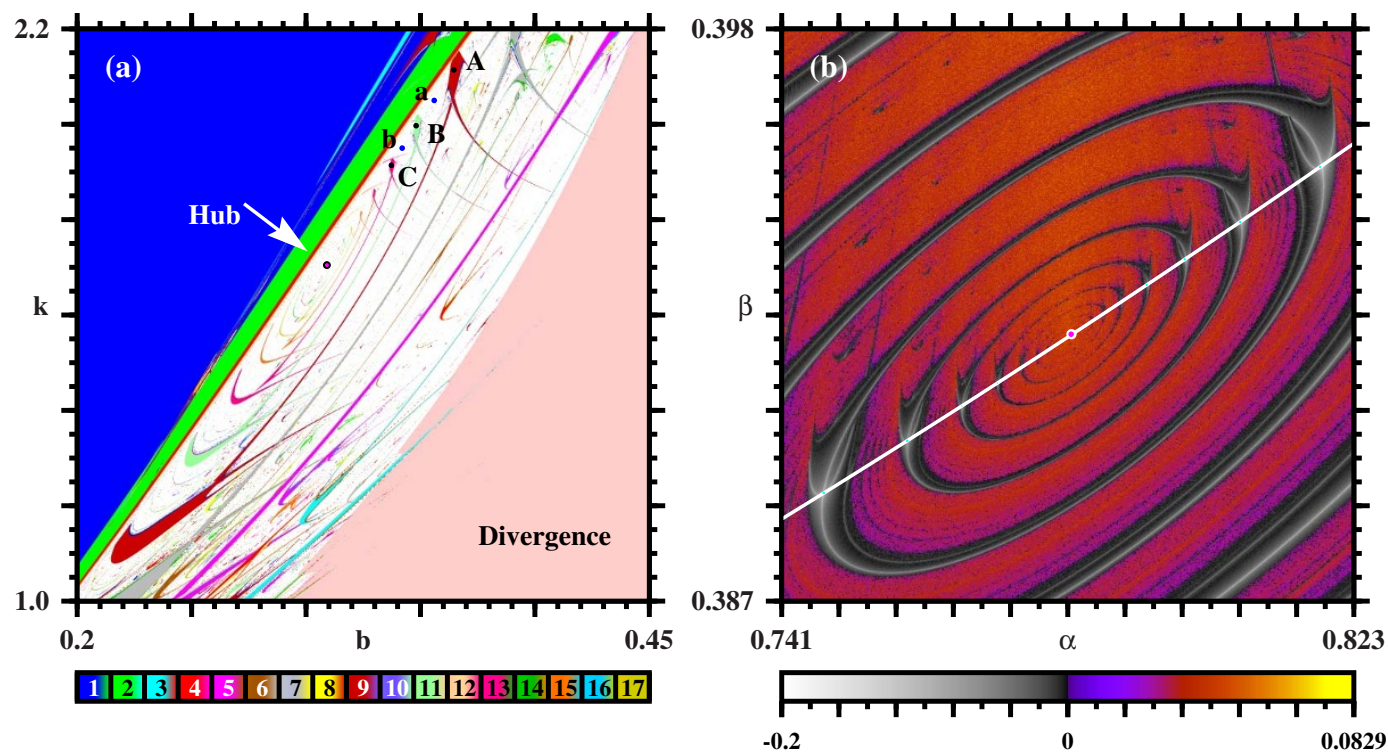

Figure 5 Comparison of structural details between (a) the new discontinuous spiral composed of the terminations shown in Fig. [6](a), and (b) an example of the known continuous spiral, composed of the "shrimp" in Fig. [6](b), here displaying Lyapunov exponents for a model of an abstract chemical reaction ${ }^{3}$. Both spirals have a rather distinct structure and cannot be deformed one into the other.

chaos from regularity. In other words, with the hindsight provided by the diagram of Lyapunov exponents, it is easy to recognize that in Fig. [3](a) blue represents periodic solutions while the yellow-red colors represent chaotic oscillations.

Comparing the stability diagrams in Figs. [3] (a) and [3](b) one can recognize the presence of a small shift between the parameter scales: This happens because in the experiment $b$ has to compensate all losses in the circuit, due mainly to the internal series resistance of the inductor. This means using $b-(26 \Omega) /(156 \Omega)$ (see Fig. [1]) as the effective coefficient. Other losses, like e.g. the ones due to magnetization of the ferrite core, are difficult to model and have not been explicitly included, leading to small additional shifts in $b$. Further, experimentally it was difficult to set $\omega$ to the desired value 0.5 by tuning a capacitor. Additionally, $\omega$ may change with frequency because of the frequency dependence of the dielectric in the $13.8 \mathrm{nF}$ capacitor in Fig. [1]. Fortunately, from Eqs. (2) we see that $k z=k \dot{x}-k \dot{z} / \omega$, showing that $k$ can partially compensate shifts in $\omega$. Despite the aforementioned difficulties, the robust and unambiguous message from Fig. [3] is that there is excellent agreement between experiments and simulations to very fine details, as corroborated by the comparisons in Fig. [4].

Figure [3] displays a number of interesting and hitherto unknown features. The discontinuous spiral consists of a discrete alternation of two types of periodic phases, each one characterized by oscillations having similar wave patterns (shown in panels $A, B, C$ of Fig. [3] and an ever increasing number of maxima as one moves along the spiral towards the central focal point. The upper right corner of both panels in Fig. [3] display sequences of certain "fish-like" structures with thin and very long tails. In contrast, the lower left corner shows an alternation of a composite involving crescent-shaped boomerang-like forms together with cuspidal "harpoons" which also have long tails. These two types of structures are shown magnified in Fig. [4], which also presents a comparison between measured and simulated phases.

How do the discontinuous spirals reported here compare with the known continuous spirals from the literature and illustrated in Fig. [5](b)? Is it possible to isomorphically deform continuous and discontinuous spirals into one another? Are the terminations forming the discontinuous spirals just distorted shrimps projected from an exquisite angle in the control parameter space? The answer is simple: Both spirals are intrinsically distinct objects that are not isomorphic, i.e. cannot be deformed into one another. In the isospike diagram seen in Fig. [5] (a), colors represent the number of maxima observed in one period of $x(t)$, while white is used to represent chaotic oscillations. This figure shows that the number of peaks in one period of $x(t)$ increases steadily by 2 towards the accumulation focal point. The spirals in Fig. [5] arise from distinct bifurcations ${ }^{10}$. This can be understood from Fig. [5] where we compare the familiar shrimps ${ }^{1,18-20}$ that wind to form continuous spirals, with the two sets of terminations involved in the discontinuous spiral. Although legs can get very elongated and thin and slightly change their thickness upon changes of parameters, there is no way to make the distinct terminations to coincide, nor to avoid the characteristic chaotic gaps present in the discontinuous spiral ${ }^{10}$.

The boomerang-like terminations do not contain any cusps inside them and, therefore, they cannot be elongated or distorted shrimps. Both terminations composing the discontinuous spiral can be found abundantly as isolated structures (i.e. not forming spirals) in many maps like, e.g. the two-dimensional quadratic Hénon map ${ }^{1,18}$ $(u, v) \mapsto\left(a-u^{2}+b v, u\right)$, or in the characteristic pair of one-dimensional cubic maps. Fig. [6] illustrates fish-like, boomerang-like, and cusp-like terminations of period-10 generated by the Hénon map. The pink background denotes parameters leading to divergent solutions. As indicated by the colorbar and numbers in the figure, the main bodies of the terminations have period 10. Similar terminations but with period 14 exist inside the box in the lower left corner. The characteristic terminations of the discontinuous spiral have been studied in detail both in the real and in the complex plane $e^{20}$.

The possibility of studying the structural organization of discontinuous spirals using low-dimensional maps is important for two reasons. First, maps allow one to avoid the complications associated with the integration of differential equations. Second, one-dimensional maps allow one to follow the self-similar organization in terms of the superstable loci of the maps, a concept with no equivalent in flows or in multidimensional maps. After knowing that the peculiar terminations forming discontinuous spirals can be well reproduced with simple maps, an interesting open question is to find out what sort of mechanism is necessary to bend terminations in order to form long spirals. Furthermore, since models contain a myriad of parameters, it would be interesting to divine a working model containing spirals with the least distortion and compression possible. This 

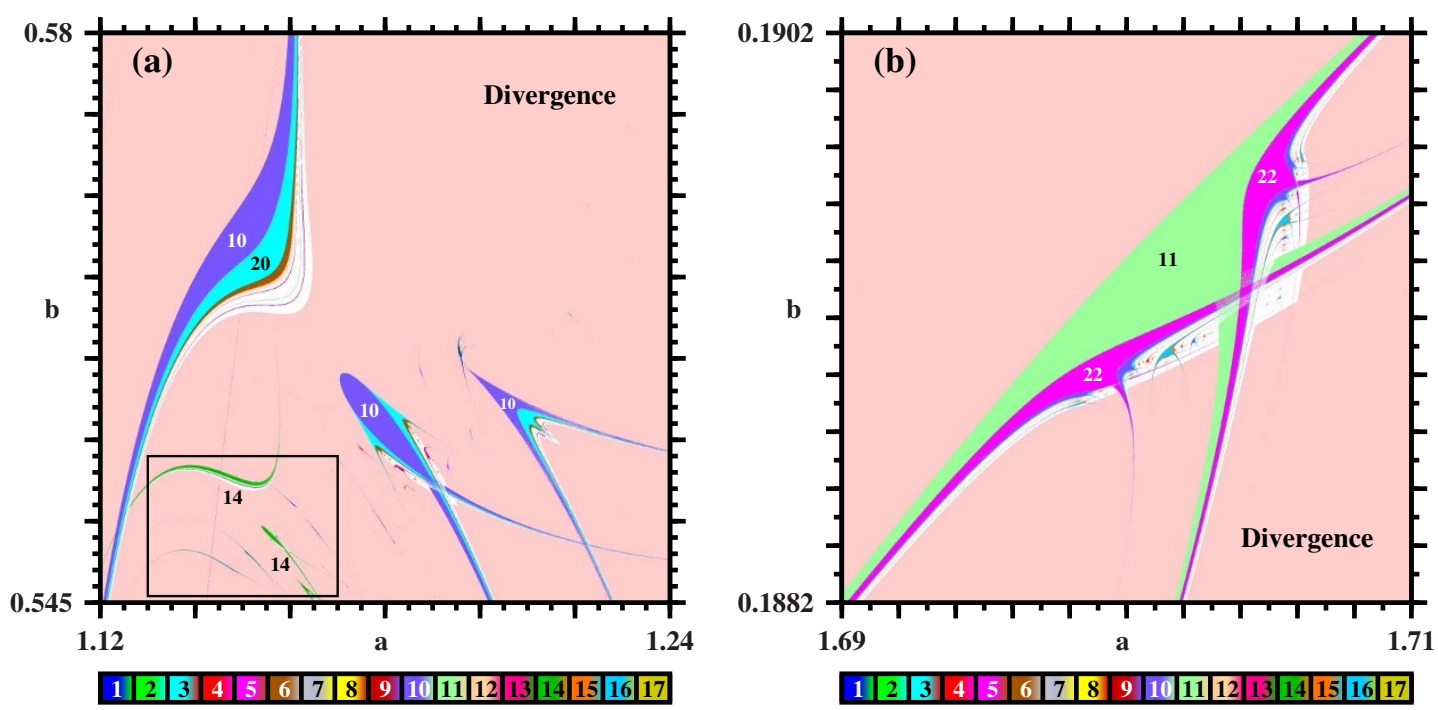

Figure $6 \mid$ (a) Prototype terminations which form the discontinuous spiral ${ }^{20}$, (b) Prototype of the "shrimp" structure ${ }^{1,18,19}$ found at every turn of any continuous spiral. Numbers refer to periods, white marks chaos. These forms are not isomorphic to each other ${ }^{10}$. See text.

would avoid the idiosyncrasies of using planar projections to represent complicated multidimensional parameter surfaces in control space.

Of relevance for applications is that, while continuous spirals allow smooth navigation towards the focal hub without the need of ever crossing the omnipresent chaotic phases surrounding the spiral, analogous navigation along discontinuous spirals forces one to repeatedly move across chaotic phases while whirling to or from the focal hub. The monotonous regularity of such alternation between chaotic and periodic phases seems to allow one to anticipate where and how big are the intervals between phases. We have not attempted to characterize such discontinuities here. Note that the parameter volume of the chaotic phase connected with discontinuous spirals seems to be considerably larger than the corresponding phase connected with continuous spirals.

\section{Discussion}

In summary, we reported the experimental discovery and numerical corroboration of a remarkable discontinuous spiral in the control space of an electronic circuit. Our results show that, in addition to the familiar class of continuous spirals, periodic oscillations may also form a new class of discontinuous spirals in stability diagrams. Discontinuous spirals also whirl around an exceptional focal point that organizes very orderly the dynamics over wide portions of the control parameter space. Although there is no mathematical theory yet to predict the location of focal hubs and their spirals, our work shows that they can be located both in the laboratory and with numerical simulations. Computer models mirroring real-life experiments like the one described here have become crucial for most advances in the understanding of complex phenomena affecting the control parameter space globally.

The circuit studied here can be considered as a paradigm for a class of phenomena governed by nonlinear differential equations, whose dynamics should present similar discontinuous features. Knowledge of the location and structure of spirals is of use for controlling and/or safely guiding system in control space. The understanding of the farreaching distribution of spiral phases emanating from periodicity hubs is a challenging theoretical matter. In some flows, periodicity hubs are known to be connected with Shilnikov's scenario of homoclinic bifurcations ${ }^{5-7}$. However, it is equally known that such homoclinic bifurcation scenario is not a necessary condition for the existence of hubs and spirals ${ }^{16}$. In fact, periodicity hubs with their continuous spirals are instantiations of a much more general phenomenon which is observed also in situations transcending Shilnikov's scenario and involving no homoclinic connections at all ${ }^{5,16}$. In sharp contrast with its continuous counterpart, nothing is presently known theoretically about discontinuous spirals. We hope their discovery now to motivate the determination of the mathematical conditions underlying their genesis. The investigation of spirals should also help to shed light on the rather difficult question of classifying in how many different ways can we expect stability islands to accumulate in phase diagrams of nonlinear oscillators and, more generically, in dynamical systems.

\section{Methods}

Experimental setup. All operational amplifiers used in our experiment (Fig. [1]) are fast, precise and of low-noise (AD8676), the resistors are of low temperature coefficient $(<5 \mathrm{ppm} / \mathrm{K})$ and of low tolerance, $0.1 \%$, except for the resistors in the diode network, and $R_{L}$, the internal resistance of the copper wire wound coil. The diodes are of the Schottky type. Since the forward voltages on the diodes and $R_{L}$ are quite temperature sensitive, the whole setup was placed inside a thermally stabilized box that allowed maintaining the air temperature constant at $25.0 \pm 0.05^{\circ} \mathrm{C}$ during the measurement. The voltages on the points $x$ and $z$ in Fig. [1] were recorded using a standard PC with integrated analog-to-digital converter. Our measurements are robust against slight changes of the specific parameter values used in the circuit.

Experimental measurements. For each individual parameter pair we started by, during about $10 \mathrm{~ms}$, setting to zero the digitally controlled attenuator responsible for $b$ to allow any spurious oscillation to decay. After selecting each value of $b$ and $k$, we waited for about $0.2 \mathrm{~s}$ to allow transients to die out. The voltages $x$ and $z$ were then sampled with $192 \mathrm{ksps}$ for $0.5 \mathrm{~s}$ and converted into pairs of data points representing the original $x$ and $z$ voltages over time. With the $(x, z)$ so obtained we computed a twodimensional histogram with a binning of $500 \times 500$ and a range of $\pm 3 \mathrm{~V}$ counts per axis. After this, the number of bins containing non-zero values was evaluated. This simple approach quantifies the occupied fraction of the phase-space and may be measured either in pixels or in volts squared. The acquisition of a single sample in parameter space takes about 1 second. The above procedure was repeated for every point of the $(b, k)$ parameter grid. The values quantifying the occupied phase-space for a range of parameters were then normalized and used to build a graphical representation of the data such that the individual values contained in a matrix of points are represented as colors. Each value quantifying the occupied fraction of phase-space is represented by a different color, ranging from dark blue for low occupation fractions up to dark red for high occupation. An essentially identical phase diagram (not shown here) was measured using the following quite different method of recording oscillations. In this second procedure, we counted the number of peaks (local maxima) in each $x(t)$ signal, binned them into 1000 bins, counted the distinct peaks of the histogram so obtained, and used this count as a color index to build the phase diagram. Clearly, the idea behind this color index is to associate low number of peaks to periodic solutions and larger number of peaks to chaotic behaviors.

Computational methods. Lyapunov exponents were obtained by solving Eqs. (2) numerically using a standard fourth-order Runge-Kutta algorithm with fixed-step, 
$h=0.0045$, over a high-resolution mesh consisting of $2400 \times 2400=5.76 \times 10^{6}$ equally spaced points. For each point $(b, k)$ of the mesh, we computed the three Lyapunov exponents by always starting numerical integrations from a fixed arbitrarily chosen initial condition: $(x, y, z)=(0.02,0.1,0.11)$. The first $10^{5}$ time-steps were discarded as transient time needed to reach the final attractor. The subsequent 5 $\times 10^{6}$ iterations were then used to compute the Lyapunov spectrum of the oscillator. In Figs. [3](a), [5](a), and [6], the pink background denotes parameter values leading to divergent solutions. As indicated by the colorbar in Fig. [5](a), a palette of 17 colors is used to represent "modulo 17" the number of peaks (maxima) in one period of the oscillations of $x(t)$. Similarly, in Fig. [6], the periodicity is plotted modulo 17. The isospike diagram shown in Fig. [5] (a) was obtained after computing Lyapunov exponents by recording up to 800 extrema (maxima and minima) of the time series of $x(t)$ together with the instant when they occur, and recording repetitions of the maxima. The computation of high-resolution stability diagrams for several millions of points is numerically a quite demanding task that we performed with the help of 1536 high-performance processors of a SGI Altix cluster with a theoretical peak performance of 16 Tflops.

1. Lorenz, E. N. Compound windows of the Hénon map. Physica D 237, 1689-1704 (2008).

2. Francke, R. E., Pöschel, T. \& Gallas, J. A. C. Zig-zag networks of self-excited periodic oscillations in a tunnel diode and a fiber-ring laser. Phys. Rev. E 87, 042907 (2013).

3. Gallas, J. A. C. The structure of infinite periodic and chaotic hub cascades in phase diagrams of simple autonomous flows. Int. J. Bif. Chaos 20, 197-211 (2010).

4. Freire, J. G., Cabeza, C., Marti, A., Pöschel, T. \& Gallas, J. A. C. Antiperiodic oscillations. Sci. Rep. 3, 01958 (2013).

5. Vitolo, R., Glendinning, P. \& Gallas, J. A. C. Global structure of periodicity hubs in Lyapunov phase diagrams of dissipative flows. Phys. Rev. E 84, 016216 (2011).

6. Barrio, R., Blesa, F., Serrano, S. \& Shilnikov, A. Global organization of spiral structures in biparameter space of dissipative systems with Shilnikov saddle-foci. Phys. Rev. E 84, 035201(R) (2011).

7. Barrio, R., Blesa, F. \& Serrano, S. Topological changes in periodicity hubs of dissipative systems. Phys. Rev. Lett. 108, 214102 (2012).

8. Shilnikov, A., Shilnikov, L. P. \& Barrio, R. Symbolic dynamics and spiral structures due to the saddle-focus bifurcation, in "Chaos, CNN, Memristors and Beyond", Adamatzky, A. \& Chen, G. (eds.), 428-439 (World Scientific, Singapore, 2013).

9. Junges, L., Pöschel, T. \& Gallas, J. A. C. Characterization of the stability of semiconductor lasers with delayed feedback according to the Lang-Kobayashi model. Eur. Phys. J. D 67, 149 (2013)

10. Arnold, V. I. Lectures on bifurcations in versal families. Russ. Math. Surv. 27, 54-123 (1972)

11. Unlocking Dynamical Diversity: Optical Feedback Effects on Semiconductor Lasers Kane, D. M. \& Shore, K. A. (eds.), (Wiley, New York, 2005).

12. Bursting, The Genesis of Rhythm in the Nervous System Coombes, S. \& Bressloff, P. C. (eds.), (World Scientific, Singapore, 2005).
13. Kautz, R. Chaos: The Science of Predictable Random Motion (Oxford University Press, Oxford, 2011).

14. Lindberg, E. et al. Autonomous third-order Duffing-Holmes type chaotic oscillator, pages 663-666, in IEEE Proceedings of the European Conference on Circuit Theory and Design, held in Antalya, Turkey (23-27 Aug. 2009). DOI: 10.1109/ECCTD.2009.5275062.

15. Tamaševičius, A. et al. Autonomous Duffing-Holmes type chaotic oscillator. Electronics and Electrical Engineering T170, 43-46 (2009).

16. Freire, J. G. \& Gallas, J. A. C. Non-Shilnikov cascades of spikes and hubs in a semiconductor laser with optoelectronic feedback. Phys. Rev. E 82, 037202 (2010)

17. Marino, F. et al. Mixed-mode oscillations via canard explosions in light-emitting diodes with optoelectronic feedback. Phys. Rev. E 84, 047201 (2011).

18. Gallas, J. A. C. Dissecting shrimps: results for some one-dimensional physical systems. Physica A 202, 196-223 (1994).

19. Façanha, W., Oldeman, B. \& Glass, L. Bifurcation structures in two-dimensional maps: The endoskeletons of shrimps. Phys. Lett. A 377, 1264-1268 (2013).

20. Endler, A. \& Gallas, J. A. C. Mandelbrot-like sets in dynamical systems with no critical points. Comptes Rendus Academie des Sciences, Mathematique (Paris) 342, 681-684 (2006).

\section{Acknowledgments}

J.G.F. was supported by FCT, Portugal through the Post-Doctoral grant SFRH/BPD/43608/ 2008. This work was supported by the Deutsche Forschungsgemeinschaft through the Cluster of Excellence Engineering of Advanced Materials. All bitmaps were computed at the CESUP-UFRGS clusters, in Porto Alegre, Brazil. JACG thanks support from CNPq, Brazil.

\section{Author contributions}

A.S., E.L., T.P. and J.A.C.G. conceived the experiments. A.S. designed and performed the experiments. J.G.F. and J.A.C.G. performed the simulations. J.A.C.G. wrote the main manuscript. All authors discussed the results and reviewed the manuscript.

\section{Additional information}

Competing financial interests: The authors declare no competing financial interests.

How to cite this article: Sack, A., Freire, J.G., Lindberg, E., Pöschel, T. \& Gallas, J.A.C Discontinuous Spirals of Stable Periodic Oscillations. Sci. Rep. 3, 3350; DOI:10.1038/ srep03350 (2013)

(c) (i) $\odot$ This work is licensed under a Creative Commons AttributionNonCommercial-NoDerivs 3.0 Unported license. To view a copy of this license, visit http://creativecommons.org/licenses/by-nc-nd/3.0 